\title{
Research on the Fusion of AR Technology in Home Space Design
}

\author{
Wen-Bo ZHAO
}

\author{
No. $1 \mathrm{Ha}$ nan road nineteen, Ha nan industrial town, Ping Fang District, Harbin, China \\ 75646@qq.com
}

Keywords: AR technology, augmented reality, home space design, smart home.

\begin{abstract}
AR technology is an advanced home automation system, which saves energy and time of design. How to reasonably use AR technology to solve the problems of long design cycle and high investment in the current home design industry, and focus on improving the quality of home space design from the perspective of human-computer interaction. Interdisciplinary knowledge is used to combine the cutting-edge AR technology in this subject with the home space design industry, aiming to improve the quality of home space design by using the latest scientific and technological means, and systematically analyze the collected data, and predict the development of the home industry. Using AR technology to create a more dynamic home space for mankind, but also can reduce the consumption and waste of design resources, truly achieve sustainable development.
\end{abstract}

\section{Introduction}

Use italic for emphasizing a word or phrase. Does not use boldface typing or capital letters except for section headings (cf. remarks on section headings, below). Augmented Reality (AR) is a new type of human-computer interaction technology, which puts the virtual world on the screen and interacts with the real world, calculates the position and angle of the camera image in real time and adds the corresponding image. AR technology is a new technology that seamlessly integrates real world information and virtual world information. It will be difficult to experience physical information in real space and time (vision, smell, touch, taste, etc.) virtual to computer software, through computer technology, simulation, superposition, the virtual information will be applied to the real world, human senses perceived, so as to achieve beyond the real sensory experience. Real environment and virtual objects are superimposed on the same screen or space in real time. It gives people a surreal immersion experience. AR technology includes multimedia, 3D modeling, real-time video display and control, multi-sensor fusion, real-time tracking and registration, scene fusion and other ew technologies and means. AR technology provides information different from human perception.

\section{Application Status of AR Technology}

In recent years, AR technology has been widely concerned and enjoyed by people, and has great prospects for development. It is precisely because more and more AR technologies have been developed that AR has been integrated into our lives. It has changed our way of life in the past and our understanding and cognition of the original world. Nowadays, AR technology is widely used in military, medical, educational and other industries. At present, the application in this field abroad has been more extensive, but in our country, affected by the number of employees, development cycle, development costs and user awareness, has not been widely 
developed and applied. But as far as AR technology is concerned, it has more abundant and intuitive information, and has more authentic characteristics in interactive experience. It can be said that AR technology is an important trend in the future development of science and technology. AR technology is an advanced family automation system, it can save our energy and time. For smart home, we think AR technology is an important way of human-machine interaction in smart home.

Through the use of man-machine, voice, motion capture, somatosensory interaction and other ways, can greatly improve the efficiency and quality of family life. Using the least communication, the least operation and the simplest operation to make the system run is not only the core of human-computer interaction, but also the goal of AR technology.

\section{The Development and Influence of AR technology on Home Space Design}

The application of AR technology in home space design has attracted the attention of the interior design community. However, due to the lack of technical level and hardware facilities, the application effect of AR technology is not ideal. Because the hardware facilities and technical level are difficult to reach the standard, there is a certain gap between the design results and the scene presented by AR technology. But with the improvement of AR technology and the improvement of hardware facilities, the problems will be constantly improved. Designers and relevant technicians will also persevere in the skilled application of AR technology, and AR technology will bring a new technological revolution to the design of home space. The application of AR technology is not just a dream. Using AR technology to create a more dynamic home space for human beings, to provide people with more exciting home space experience, but also can reduce the consumption and waste of indoor design resources, truly achieve sustainable development.

\section{Advantages of AR technology in Home Space Design}

\section{Sensor Scene Fusion}

Multi-sensor fusion technology not only makes up for the shortage of single sensor in automatic remote sensing technology, but also applies to AR technology. Through multi-sensor technology to show the real scene in the home, but also through multi-stereo sound pronunciation, touch screen control space furniture, appliances, soft packaging and other virtual objects to simulate the real space, to give customers a surreal immersion experience.

\section{Easy to Use and Easy to Implement}

AR technology allows users to see computer-generated images in realistic scenes through the seamless connection between virtual images and real images. With the continuous development and improvement of related technologies, AR technology can create a visual effect of false and confusing. People can use AR-related software on mobile phones and computers. Compared with VR technology which requires special helmet and handle, AR technology is more economical and easier to implement. 


\section{Improve Efficiency and Facilitate Communication}

Software such as CAD and 3ds Max, who expresses design techniques, can neither modify and adjust drawings in real time nor display design works quickly. The process of production modification is complex and the modification cycle is long, which makes the cost of production high. To a certain extent, it restricts the designer's creation and ideas. There will be some deviations when we display design works. AR technology can provide real-time modeling information, rich texture materials, and quickly adjust changes in the scene. Designers add design creation to the real scene, maximize the restoration of the designer's ideas, and complete presentation of the designer's ideas, while improving the efficiency and quality of design.

\section{AR Integration in Home Space Design}

AR technology in the field of home space design applications, so that designers more intuitive and more detailed design of space details. Let the designer experience every real feeling of design in the virtual space, even touch the texture of furniture in the space, feel the scale of space. Plane layout, material selection, furniture layout and other aspects of the design process to provide designers with more accurate data, design form and real space combined to provide customers with more intuitive data, is conducive to communication between designers and customers.

\section{Basic application of AR Technology}

\section{Basic Modeling}

Three-dimensional modeling is the core part of traditional rendering and AR technology, in which real environment modeling with high degree of simulation is a unique important part of AR technology. According to the actual size data, the application software is used to create and render the corresponding structures, furniture and soft decorations in the real space environment. After modeling and rendering, the interactive information is established by computer interactive software, and the interactive information is transmitted to the corresponding equipment. Finally, the interaction between virtual model and real space is completed.

\section{Synchronous Data}

After three-dimensional modeling is completed, it is necessary to create a full-view and real-time dynamic virtual scene, use 3D game engine to complete man-machine synchronization, use camera equipment, accelerator and other sensors to feedback user information to the virtual scene, each action of the user can be quickly synchronized to the screen and can be in the virtual scene. Move in the middle. The data model and the multimedia effects are projected into people's eyes, enabling the experiencer to manipulate the virtual elements in real space, and using AR technology to display the home design scheme more comprehensively and intuitively.

\section{A Full Range of Design Proposals}

The traditional home design is to convey the designer's creativity and ideas through the construction drawing and the rendering. In the later period, some designers used VR technology and panoramic effects to display their designs. These techniques require designers to spend a lot of drawing time to draw, communication programs to adjust and modify the waste of a lot of time and drawing costs. However, the application of AR technology has solved these problems. 
By using AR technology, the designer directly outputs the interactive operation between the virtual scene and the real scene after three-dimensional modeling. Designers and customers can also adjust and modify the details of the scene through virtual devices, and deal with every design node in an all-round way. The use of AR technology saves designer's drawing time and improves the designer's creative and thinking quality.

\section{Summary}

As a new technology, AR technology has unlimited development prospects in all industries. At present, the application of AR technology in home space design is not ideal, but its development prospect is considerable. AR technology can give us the same visual, auditory and tactile perception as the real environment. The efficiency and customer satisfaction of home space design will be greatly improved. Whether in the field of home space design, or in every field of our daily life. AR technology will subvert our life on the basis of the development of virtual reality. Although AR technology in the field of home space design is still in its infancy, but through the strong advantages of AR technology itself, can make more home space design practitioners concerned about and make full use of AR technology. AR technology has great practical significance and application value for the home space design industry. I believe that AR technology will provide new impetus for the development of the whole household industry.

\section{Acknowledgement}

Thank you for giving this opportunity to present a paper at the 4th International Conference on Educational Science and Human Development (ESHD 2018). Thank the school leaders for their support. This paper is one of the research results of Heilongjiang Oriental College's school-level scientific research project "Application Research of Virtual Reality (VR) in Harbin Home Design". Project number: HDFHX170216. Zhao Wenbo, 1982.11.14, female, lecturer, research direction: Environmental Design. Studio: Department of art and design, East University of Heilongjiang

\section{References}

[1] CAI Jinling. Augmented reality application research in onlineeducation [J]. Computer knowledge and technology, 2017, 13(6):148-149.

[2] LIN Yi, CHEN Jing, LIU Yue, et al. User experience design ofVR-AR hybrid mobile browsing system based on mental model [J]. Chinese journal of computers, 2015, 38(2):408422 .

[3] XU Lingying. Necessity and feasibility analysis for VR/AR tech-nology embedded in scitech periodical publishing [J]. Chinesejournal of scientific and technical periodicals, 2017, 28(6):532-537.

[4] YU Ri-ji, CAI Min, JIANG Shuai. Design and Appli-cation of Museum Cultural Education Experience Sys-tem Based on Mobile Terminal and AR Technology [J]. China Electrochemical Education, 2017(3): 31-35. 
[5] Zhou Yong. Application of VR and AR Technology in Interior Design [D]. Southeast University, 2017.

[6] Wang Xiaohui. Design of healthy high-rise residential buildings under sustainable concept [J]. Engineering technology research, 2018 (03): 219-220.

[7] Hou Ying, Xu Wei. Summary of augmented reality technology [J]. Computer measurement and control, 2017, 25 (2): 1-7, 22.

[8] Ding Nan, Wang Yamin. Application of Virtual Reality in Education: Advantages and Challenges [J].Modern Educational Technology, 2017, 27 (2): 19-25.

[9] Zhang Fengjun, Dai Guozhong, Peng Xiaolan.A review of human-computer interaction in virtual reality [J].China Science: Information Science, 2016, 46(12): 1711-1736.

[10]Wang Lili, Chen Jinying, Feng Guangnan. Opportunities and Challenges of VR/AR Technology [J].Communications and Information Technology, 2016 (6): 64-65, 76. 